Research Paper

\title{
Transplantation of DI5A-Expressing Glial-Restricted- Precursor-Derived Astrocytes Improves Anatomical and Locomotor Recovery after Spinal Cord Injury
}

\author{
Chunling Fan ${ }^{1,2}$, Yiyan Zheng2, Xiaoxin Cheng2 ${ }^{2}$, Xiangbei Qi' ${ }^{2,3}$, Ping Bu², Xuegang Luo ${ }^{1}$, Dong H. Kim², \\ Qilin $\mathrm{CaO}^{2 \mathrm{M}}$ \\ 1. Department of Anatomy and Neurobiology, Central South University Xianya Medical School, Changsha, Hunan 410011, P.R. China. \\ 2. The Vivian L Smith Department of Neurosurgery, UT Medical School at Houston, Houston, TX 77030. \\ 3. Department of Orthopedic Surgery, the Third Affiliated Hospital, Hebei Medical University, Hebei050051, P.R.China.
}

$\triangle$ Corresponding author: Dr. Qilin Cao, Department of Neurosurgery, UT Medical School at Houston, 6341 Fannin Street, MSE R158, Houston, TX 77030, Tel: (713) 500-3445 / Fax: (713) 500-7787, email:qi-lin.cao@uth.tmc.edu.

( ) Ivyspring International Publisher. This is an open-access article distributed under the terms of the Creative Commons License (http://creativecommons.org/ licenses/by-nc-nd/3.0/). Reproduction is permitted for personal, noncommercial use, provided that the article is in whole, unmodified, and properly cited.

Received: 2012.11.29; Accepted: 2012.12.19; Published: 2012.12.22

\begin{abstract}
The transplantation of neural stem/progenitor cells is a promising therapeutic strategy for spinal cord injury (SCl). In this study, we tested whether combination of neurotrophic factors and transplantation of glial-restricted precursor (GRPs)-derived astrocytes (GDAs) could decrease the injury and promote functional recovery after $\mathrm{SCl}$. We developed a protocol to quickly produce a sufficiently large, homogenous population of young astrocytes from GRPs, the earliest arising progenitor cell population restricted to the generation of glia. GDAs expressed the axonal regeneration promoting substrates, laminin and fibronectin, but not the inhibitory chondroitin sulfate proteoglycans (CSPGs). Importantly, GDAs or its conditioned medium promoted the neurite outgrowth of dorsal root ganglion neurons in vitro. GDAs were infected with retroviruses expressing EGFP or multi-neurotrophin DI5A and transplanted into the contused adult thoracic spinal cord at 8 days post-injury. Eight weeks after transplantation, the grafted GDAs survived and integrated into the injured spinal cord. Grafted GDAs expressed GFAP, suggesting they remained astrocyte lineage in the injured spinal cord. But it did not express CSPG. Robust axonal regeneration along the grafted GDAs was observed. Furthermore, transplantation of DI5A-GDAs significantly increased the spared white matter and decreased the injury size compared to other control groups. More importantly, transplantation of DI5A-GDAs significantly improved the locomotion function recovery shown by BBB locomotion scores and Tredscan footprint analyses. However, this combinatorial strategy did not enhance the aberrant synaptic connectivity of pain afferents, nor did it exacerbate posttraumatic neuropathic pain. These results demonstrate that transplantation of DI5A-expressing GDAs promotes anatomical and locomotion recovery after $\mathrm{SCl}$, suggesting it may be an effective therapeutic approach for $\mathrm{SCl}$.
\end{abstract}

Key words: astrocytes, oligodendrocyte, transplantation, spinal cord injury, remyelination.

\section{Introduction}

Despite extensive research, clinical advancements, and improved rehabilitation strategies, spinal cord injury (SCI) continues to be a major cause of disability and mortality. Unfortunately, no treatments are currently available to promote significant functional recovery. Therefore, new therapeutic strategies are urgently needed. Stem cells have shown great therapeutic potentials for SCI repair in several ex- 
perimental models and may represent one of the effective novel therapies. Neural stem cells (NSCs) [1-3] or oligodendrocyte precursor cells (OPC) [4-8] differentiate into mature oligodendrocytes (OL), increase remyelination and enhance the functional recovery after transplantation into the injured spinal cord. Grafted NSCs or neuronal progenitor cells have also shown to differentiate into neurons and potentially replace the lost neurons after SCI [9-11]. In addition to the neuronal and OL replacement, NSCs or neural progenitor cells could also decrease the injury and promote constitutive repair by modifying the injury microenvironment. Stem cell and progenitor cells could secrete neurotrophic factors which are known to alter injury and disease pathogenesis. For example, the transplanted human NSCs constitutively secrete nerve growth factor, brain-derived neurotrophic factor (BDNF) and glial-derived neurotrophic factor (GDNF) which could promote the growth of host axons after SCI [12]. In addition to promote remyelination, human embryonic stem cells (hESCs)-derived OPCs also express hepatocyte growth factor, transforming growth factor $b-2$ and BDNF [13], which may contribute to the functional recovery after SCI following transplantation $[4 ; 5 ; 7]$. Although grafted human ESC-derived motor neuron progenitor cells fail to differentiate into mature neurons for neuronal replacement, they decrease the injury size and functional deficits after SCI following transplantation likely by secreting multiple neurotrophins such as neurotrophin-3 (NT-3), neurotrophin-4 [14]. In additional to the trophic support, the stem cells or progenitor cells can also provide substrates or decrease the inhibitors to promote the axonal regeneration after SCI. For example, young astrocytes derived from glial-restricted precursor cells (GRPs) decrease astrogliosis and CSPG expression and, importantly, promote axonal regeneration after transplantation following SCI $[15 ; 16]$. GRPs or NSCs differentiate mainly into astrocytes to change the microenvironment to promote neurogenesis after transplantation into hippocampus of aged rat [17]. Thus, transplanted stem cell derivates can modify the injured environment by providing survival factors, guidance molecules, or cues for proliferation and differentiation of endogenous stem and progenitor cells.

Neurotrophins play important roles in axonal regeneration and plasticity in developing and adult animals following SCI [18-20]. Neurotrophin-mediated regeneration of specific supraspinal and sensory pathways has been well documented [21]. Particularly, neurotrophins NT-3 exerts strong and preferential effects on regeneration of injured axons in CST [22-24] and in DC [25-27]. BDNF signif- icantly promotes the regeneration of rubrospinal and supraspinal tracts after SCI [28-30]. Additionally, neurotrophins also play important roles on remyelination after CNS injury. For example, transplantation of fibroblasts genetically modified to express NT3 or BDNF in the injured spinal cord increases the proliferation of OPCs and the myelination of regenerating and/or spared axons [18]. Our previous study also showed that transplantation of GRPs genetically over-expressing D15A, a novel neurotrophin with both NT3 and BDNF activities [31], promoted the functional recovery of electrophysiological conduction and locomotor [6]. However, it still remains to determine whether the functional recovery comes from OL remyelination by grafted D15A-GRPs or its neurotrophin effects, or both. Grafted 15A-GRPs secrete D15A into the injured spinal cord, and they also differentiate into mature OLs which remyelinate the demyelinated axons. Importantly, expression of D15A further increase the OL differentiation of grafted GRPs. It will be difficult to distinguish the contributions of OL differentiation and remyelination and neurotrophic effects of grafted D15A-GRPs to functional recovery.

In this study, we induced GRPs to differentiate into astrocytes in vitro before transplantation and tested the therapeutic potential of GRP-derived astrocytes (GDAs) after SCI. GDAs lost its potential to differentiate into OLs both in vitro and in vivo. Thus, functional recovery after GDA transplantation will depend on mechanisms other than graft-derived remylination. Such study combining with our previous GRP transplantation study [6] will help us understand the underlying mechanisms for functional recovery following cell transplantation. Such understanding may help us to optimize our cell transplantation-based therapies for SCI and other neurological diseases. Our results showed that the grafted GDAs survived and integrated into the host spinal cord and remained astrocyte lineage. Transplantation of GDA decreased the gliosis and formed bridge to increase the attachment of host axons in the injured epicenter. In spite of these anatomical benefits, transplantation of GDA alone did not promote significant locomotion recovery. But transplantation of D15A-GDAs significantly increased the spared white matter and decreased the injury, and, importantly, improved the locomotion function recovery after SCI. These results suggest that combination of GDAs transplantation and neurotrophin expression may be an effective therapeutic approach after SCI.

\section{Materials and Methods}

All animal care and surgical interventions were 
undertaken in strict accordance with the Public Health Service Policy on Humane Care and Use of Laboratory Animals, Guide for the Care and Use of Laboratory Animals, and with the approval of both Animal Welfare Committee and institutional biosafety committee at the University of Texas Health Science Center at Houston.

Preparation of GRPs. GRPs were immunopanned from embryonic day 14 (E14) Fischer 344 rat spinal cord using an A2B5 antibody and a protocol modified from previous studies [32;33]. Growth medium, which consisted of DMEM-F12 (Life Technologies/BRL), N2 and B27 supplements (Invitrogen, Carlsbad, CA, each at 1x), Fibroblast growth factor 2 (FGF2, $20 \mathrm{ng} / \mathrm{ml}$ ), and Platelet derived growth factor aa (PDGFaa, $10 \mathrm{ng} / \mathrm{ml}$ ), was changed every other day and growth factors (FGF2 and PDGFaa, 20 and $10 \mathrm{ng} / \mathrm{ml}$, respectively) were added daily. In all cases, an aliquot of cells was analyzed the next day to determine the efficiency of the immunopanning. Only those cell preparations in which $>95 \%$ of the bound cells expressed A2B5 were used in the experiments. After about 5 days, the cells were passaged.

To differentiate GRPs into astrocyte in vitro, the cells were plated in polyornithine/laminin-coated 24 well plates. The following day, the FGF2 was removed and $20 \mathrm{ng} / \mathrm{ml}$ bone morphogenetic protein 4 (BMP4) was added to the growth medium. Thus, differentiation medium consisted of DMEM/F12, 1x N2 and $1 \times$ B27 supplements, and BMP4 $(20 \mathrm{ng} / \mathrm{ml})$ and medium was changed every other day. After 1,3,5,7 days, the cells were fixed and immunohistochemically analyzed for their differentiated phenotypes.

Before transplantation, GRPs were infected with the LZRS retrovirus expressing either enhanced green fluorescence protein (EGFP) or D15A-EGFP. For retroviral labeling, GRPs were treated with $1 \mu \mathrm{g} / \mathrm{ml}$ polybrene for 1 hour, followed by incubation in growth medium containing LZRS-EGFP or LZRS-D15A-EGFP retrovirus for 4 hours. Routinely, about $60 \%$ cells were labeled. For GDAs transplantation, EGFP- or D15A-labeled GRPs were differentiated for 3 days in the differentiation medium with BMP4 before transplantation. Two hours before transplantation, the labeled cells were detached from the dishes using a cell lifter, collected by centrifugation at $1000 \times \mathrm{g}$ for $4 \mathrm{~min}$, and resuspended in $1 \mathrm{ml}$ culture medium. After cell count and viability assessment with trypan blue in a hemacytometer, the cell suspension was centrifuged a second time and re-suspended in a smaller volume to give a density of $5 \times 10^{4}$ viable cells $/ \mu$ l.

Co-culture of dorsal root ganglion neuron
(DRGN) and GDA. Ganglia were dissected from the entire length of the vertebral column of postnatal day 1 rats. Axons extending from the ganglia were removed under a stereoscopic microscope. The ganglia were incubated in $\mathrm{Ca}^{2+} / \mathrm{Mg}^{2+}$-free phosphate-buffered saline (PBS) containing $300 \mathrm{U} / \mathrm{ml}$ collagenase type IV (Worthington Biochemical, Lakewood, NJ, USA), $0.12 \mu \mathrm{g} / \mathrm{ml}$ DNase I and $1 \mathrm{mg} / \mathrm{ml}$ BSA for $2 \mathrm{~h}$ at $37^{\circ} \mathrm{C}$ and then rinsed with PBS to remove the enzymes. They were then incubated in PBS containing 0.25\% trypsin (Life technologies, California, USA) and $1 \mathrm{mg} / \mathrm{ml} \mathrm{BSA}$ for $15 \mathrm{~min}$ at $37^{\circ} \mathrm{C}$. After the enzymatic digestion, the cells were gently triturated with a silicon-coated Pasteur pipette and centrifuged at $1000 \mathrm{rpm}$ for $5 \mathrm{~min}$ to remove the enzymes. The cells were suspended in DMEM (Life technologies) with glucose $(4.5 \mathrm{~g} / \mathrm{l})$ and plated onto polyornithine/laminin-coated 24 well plates or on the top of GRP, or differentiated 1, 3, 5 or 7 days GDA. The cells were grown for 18 hours in neurobasal medium containing 1x B27 supplement, 1mM L-Glutamine and Nerve Growth Factor $(25 \mathrm{ng} / \mathrm{ml})$. Then the cells were fixed in $4 \%$ paraformaldehyde in $0.01 \mathrm{M}$ phosphate buffer $(\mathrm{PB})$ and processed for immunohistochemistry with first antibodies rabbit agaist neurofilament $\mathrm{M}$ (NFM, axonal marker, 1:200, Chemicon) and mouse against GFAP (astrocyte marker, 1:500, Sigma) and appropriate Fluorescence tagged secondary antibody. The longest neurite from each DRGN was measured and at least 100 DRGNs were measured in control and each co-culture. The average of the longest neurite length in each culture condition was calculated and difference among the groups was analyzed statistically using one-way ANOVA followed by Tukey's post-hoc testing.

To test the effects of GRP and GDA conditioned medium on the neurite growth of DRGNs, a whole ganglia from postnatal day 1 rats was cultured as explants in polyornithine/laminin-coated 24 well plates in neurobasal medium containing 1x B27 supplement, $1 \mathrm{mM}$ L-Glutamine and Nerve Growth Factor (25 $\mathrm{ng} / \mathrm{ml}$ ) with or without conditioned medium from GRP or GDA for 3 days. To collect the conditioned media of GRPs or GDA, 90\% confluence of GRPs or differentiated 3 day GDA were washed with DMEM/F-12 and then incubated in neurobasal medium supplemental with B27 and glutamine for 24 hours. CM was centrifuged, filtered, and concentrated 30-fold with a Centriplus 10 filter (Amicon) and stored at $-80{ }^{\circ} \mathrm{C}$ until use. The concentrated $\mathrm{CM}$ was diluted to 1:30 in fresh DRGN culture media when use. The DRG explants were stained with immune-fluorescence using NFM antibody and NFM-positive areas of DRG explants were measured 
and the difference among groups was analyzed statistically using one-way ANOVA followed by Tukey's post-hoc testing.

Construction and characterization of the D15A-LZRS retrovirus. EGFP and D15A [31] cDNAs were cloned into the LZRS retroviral vector [34]. To generate high titer virus, $\Phi$ NX cells (provided by Dr. Gary Nolan, Stanford University) were transfected using GenePorter II (Gene Therapy Systems, Inc., San Diego, CA) which routinely gave transfection efficiencies of $50-65 \%$. Selection with $2 \mu \mathrm{g} / \mathrm{ml}$ puromycin begins $48 \mathrm{hr}$ later. To harvest viral supernatant, media was changed to the appropriate serum free media without mitogens overnight and harvested the next day. We routinely obtain titers of $5 \times 10^{5}-5 \times 10^{6}$ $\mathrm{pfu} / \mathrm{ml}$.

Surgical procedures. Surgical procedures were described previously $[9 ; 35 ; 36]$. Briefly, after anesthetization with Nembutal $(50 \mathrm{mg} / \mathrm{kg}$, i.p.), adult Fischer 344 rats received a dorsal laminectomy at the $9^{\text {th }}$ thoracic vertebral level (T9) to expose the spinal cord and then a moderate to severe contusion SCI was performed by the Louisville Injury System Apparatus (LISA) at $0.8 \mathrm{~mm}$ displacement and $1.5 \mathrm{~mm} /$ second velocity [37]. At 9 days post-injury, rats were randomly assigned to four groups, which received DMEM, EGFP-GRPs, EGFP-GDAs, and D15A-GDAs, respectively. Animals were re-anesthetized as above and the laminectomy site was re-exposed. Four injections were made at $1 \mathrm{~mm}$ cranial to, caudal to and left and right of the lesion epicenter at depth $1.3 \mathrm{~mm}$ and $0.6 \mathrm{~mm}$ laterally from midline. At each site, $2 \mu \mathrm{l}$ of cell suspension or vehicle was injected through a glass micropipette with an outer diameter 50-70 $\mu \mathrm{l}$ and the tip sharp-beveled to $30-50^{\circ}$ at rate of $0.5 \mu \mathrm{l} / \mathrm{min}$ as described previously [9;36]. Thus, a total of 400,000 cells were grafted into each injured spinal cord. The animals were allowed to survive 8 weeks after transplantation.

Behavioral assessment. Open-field locomotion test using the Basso-Beattie-Bresnahan (BBB) locomotor rating scale [38] was performed once weekly for the first three weeks and then bi-weekly thereafter until 9 weeks after SCI. All the animals were coded and behavioral assessments were performed by two investigators blinded with respect to the treatment groups. The mean BBB scores were tallied by injured groups and plotted as a function of time post-injury. Changes in BBB scores over time for the four groups were analyzed using repeated measures ANOVA with the between groups factor. Differences among the groups and each group over the eight post-injury test weeks were performed using Tukey HSD post hoc t-tests.
Mechanical sensitivity test. To determine the degree of tactile sensory changes present after SCI, Von Frey hair monofilaments (VFH; Stoelting Co., Wood Dale, IL) were applied to the plantar surface of the hindpaw using a modified version of the up-down method (Chaplan et al., 1994). To ensure that all rats could withdraw their hindpaws from an unpleasant stimulus, plantar VFH was not initiated unless

hindlimb weight support recovered according to BBB testing (BBB score 10 or above). The rats were placed in an inverted acrylic glass cage $(20.25 \cdot 13.5$. $15.25 \mathrm{~cm})$ with a wire mesh bottom $(0.635 \mathrm{~cm}$ grid size) allowing access to the plantar surface of the hindpaws. Testing commenced with the 15.14-g

VFH filament applied perpendicularly at a consistent rate $\left({ }^{\star} 1 \mathrm{sec}\right)$ to the plantar surface of the hindpaw ( $1 \mathrm{~cm}$ posterior to the foot pads). When a brisk, immediate paw withdrawal occurred, the next lower VFH was applied. When no hindpaw withdrawal occurred, the next higher VFH was applied. Ten VFH applications were used, and approximately 30-60 sec separated each touch. If an animal lifted the paw, the trial was discarded and the animal was retested following the standard interstimulus interval. The tactile sensory threshold was defined as the lowest gram force needed to produce hindpaw withdrawal on at least $50 \%$ of its applications. The animals were tested before surgery and before sacrifice at 8 weeks.

Immunohistochemistry. For the histological analysis of the grafted cells, rats were anesthetized with Nembutal and perfused transcardially with 0.01 $\mathrm{M}$ phosphate buffered saline (PBS) ( $\mathrm{pH}$ 7.4), followed by $4 \%$ paraformaldehyde in $0.01 \mathrm{M}$ phosphate buffer $(\mathrm{PB})$. The spinal cord segments that received the grafts were removed, cryoprotected in 30\% sucrose buffer overnight at $4^{\circ} \mathrm{C}$ and embedded in OCT compound. Some cords were transversely sectioned at $20 \mu \mathrm{m}$ on a cryostat, while others were cut longitudinally. After blocking with $10 \%$ donkey serum in Tris buffered saline (TBS) containing $0.3 \%$ Triton X-100 (TBST) for 1 hour at room tempture (RT), the sections were incubated in TBST containing 5\% donkey serum, polyclonal chick anti-GFP (1:500, Chemicon) or polyclonal rabbit anti-neurofilament $\mathrm{M}$ (NFM, a marker for axons, 1:200, Chemicon) with one of the following monoclonal mouse antibodies: anti-glial fibrillary acidic protein (GFAP, a marker for astrocytes, 1:100, Chemicon), anti-adenomatus polyposis coli (APC, also called CC1, a marker that labels cell bodies of mature oligodendrocyte, 1:200, Pharmigen), anti-NG2 (a marker for oligodendrocyte progenitor cells, 1:200, Chemicon), or anti-nestin (a marker for undifferentiated neural stem cells or progenitor cells, 1:10, DSHB) overnight at $4^{\circ} \mathrm{C}$. After three washes of $10 \mathrm{~min}$ in TBS, 
sections were incubated in TBST containing 5\% donkey serum, donkey anti-chick FITC-conjugated $\mathrm{Fab}^{\prime}$ fragments (1:100, Jackson-ImmunoRes Lab, Baltimore, $\mathrm{MD})$, donkey anti-mouse Texas red-conjugated $\mathrm{Fab}^{\prime}$ fragments (1:200, Jackson-ImmunoRes Lab), or donkey anti-rabbit AMCA-conjugated $\mathrm{Fab}^{\prime}$ fragments (1:100, Jackson-ImmunoRes Lab) for 1 hour at RT. The sections were rinsed in TBS and cover-slipped with antifade mounting medium (Molecular Probes, Eugene, OR). Some sections were double- or triple-stained with chick anti-GFP (1:500, Chemicon) and mouse anti-GAP43 (a marker for axonal regeneration, 1:400, Sigma), or rabbit anti GFAP (1:500, Dako) or mouse anti-CSPG (1:200, Sigma) with appropriate fluorescence tagged secondary antibodies. A Nikon Eclipse TE300 inverted fluorescence microscope equipped with RT color Spot camera or a Zeiss Axiophot, LSM 510 confocal microscopy was used to capture representative images. Photomicrographs were assembled using Adobe Photoshop ${ }^{\circledR}$ and Adobe Illustrator ${ }^{\circledR}$ software.

Histological analyses. The total volume of spared white matter in the injured epicenter was determined as described previously [35]. Briefly, after the final behavioral and physiological assessments, rats were anesthetized with an overdose of Nembutal $(100 \mathrm{mg} / \mathrm{ml})$ and perfused transcardially with $0.01 \mathrm{M}$ PBS followed by $4 \%$ paraformaldehyde in PB (pH 7.4). Serial $20 \mu \mathrm{m}$ thick sections through the entire injury site were cut transversely on a cryostat. Two sets of slides (every $9^{\text {th }}$ and $10^{\text {th }}$ section, each set containing serial sections spaced $200 \mu \mathrm{m}$ apart) were stained with the iron-eriochrom cyanine R (EC) and Cresyl violet, respectively, to identify myelinated white matter and residual spared tissue. The lesion epicenter was defined as the section containing the least amount of spared white matter. White matter sparing was defined as tissue showing normal myelin appearance and density (lacking cysts, degeneration). Septae or fibrous bands of tissue observed within and/or spanning areas of cystic cavitation were not considered to represent spared tissue. The total cross-sectional area of the spinal cord and the lesion boundary were measured with an Olympus BX60 microscope attached to a Neurolucida system (Microbrightfield Inc., Colchester, VT). An unbiased estimation of the percentage of spared tissue was calculated using the Cavalieri method. The total volume of spared white and lesion area was calculated by summing their individual subvolumes [39]. Individual subvolumes of lesion area and spared tissue were calculated by multiplying the cross-sectional area (A) $x \mathrm{D}$, where $\mathrm{D}$ represents the distance between sections $(200 \mu \mathrm{m})$. The percent spared white matter and injured areas were calculated by dividing the total volume of spared white matter and lesion area by the total white matter (WM) volume and spinal cord volume, respectively. Mean values of percent lesion area and spared WM among different injured groups were calculated and statistically compared using one-way ANOVA followed by Tukey's post-hoc testing.

\section{Results}

GRP and GDA promote neurite growth in vitro. GRPs were immunopanned from E14 spinal cord with the A2B5 antibody as described previously [6;33]. All undifferentiated GRPs expressed A2B5 (Fig.1A). No GFAP expression was observed (Fig.1A). After differentiation for 1 day in the presence of BMP4, most of GRPs down-regulated their expression of A2B5 and some began to differentiate into GFAP+ astrocytes (Fig. 1B). At 3 day after differentiation, almost all GRPs differentiated into GFAP+ astrocytes (Fig. 1C). At 5 and 7 days after differentiation, expression of GFAP continued to increase in the differentiated astrocytes (Fig. 1D, E). Western blot experiments further confirmed that expression of GFAP, fibronectin and laminin was increased in the differentiated GRPs with longer differentiation time in the presence of BMP4 (E).

To directly test whether GRPs or GRP-derived astrocytes (GDAs) could promote axonal growth in vitro, the dorsal root ganglion neurons (DRGN) were isolated from postnatal day 1 rat and grown in either the PDL/laminin-coated plates or on the top of undifferentiated GRPs, or GDAs after being differentiated for 1-7 days. After culturing for 18 hours, the longest neurite from each DRGN was measured. Most DRGN cultured in the laminin-coated plates were bipolar or unipolar without secondary branch in the primary processes (Fig. 2A). However, DRGNs co-cultured with GRPs or GDAs were usually multipolar with more complex processes and secondary branches were often observed in the primary processes (Fig. 2B). The neurite length of DRGNs cultured in all cells was significantly longer than in control laminin (Fig. 2C). The neurite length cultured in GDA3d or GDA5d was significantly longer than in GRPs, or GDA1d (Fig. 2C). To further test whether promotion of neurite growth by GRPs or GDAs is mediated by soluble factors, the conditioned media (CM) from GRP or GDA3d were collected. DRG explants were cultured in neurobasal medium containing NGF $(50 \mathrm{ng} / \mathrm{ml})$ for 1 day and continue to grow in the same medium with or without GRP or GDA CMs for 2 more days. The neurite areas of DRG explants, viewed by NFM immunohistochemistry, were significantly greater in GDA or GRP CMs than in control 
medium (Fig 2D-F). These data showed that GRP, GDA or their CMs promoted neurite growth in vitro.

Survival and differentiation of grafted GRPs and GDAs in the injured spinal cord. Robust survival of grafted GRPs, GDAs or D15A-GDAs was observed in all animals. Most of grafted cells were located in the injured area and integrated in the host spared tissue (Fig. 3). The grafted cells were mainly located in the spared white matter around the cavity with some within the cavity (Fig. 3A). Extensive caudal-rostral migration was observed in all grafted GRPs, GDAs or D15A-GDAs. Some grafted cells were observed to migrate up to $1 \mathrm{~cm}$ caudally or rostrally along the central canal. The survival or migration was not significantly different among GRPs, GDAs or D15A GDAs.

Consistent with our previous study, GRPs survived and migrated extensively at eight weeks after transplantation (Fig. 4A-C). The grafted GRPs survived and migrated preferentially in white matter and sent their processes longitudinally along the axons (Fig. 4A). Some grafted GRPs differentiated into oligodendrocyte-like cells with multiple processes (Fig. 4B) and form close relationship with axons (Fig. 4C). To further examine whether these differentiated GRPs were oligodendrocyte, we double stained the GFP-labeled cells with oligodendrocyte specific marker, CC1. As shown in Figure 4D-F, some grafted GRPs differentiated into CC1-positive oligodendrocytes. More grafted GRPs differentiated into GFAP-positive astrocytes (Fig. 4G-I). No neuronal differentiation was observed (data not shown). The results were consistent with previous studies [6;40] showing that GRPs were bi-potential in vivo to differentiate into both astrocytes and oligodendrocytes.
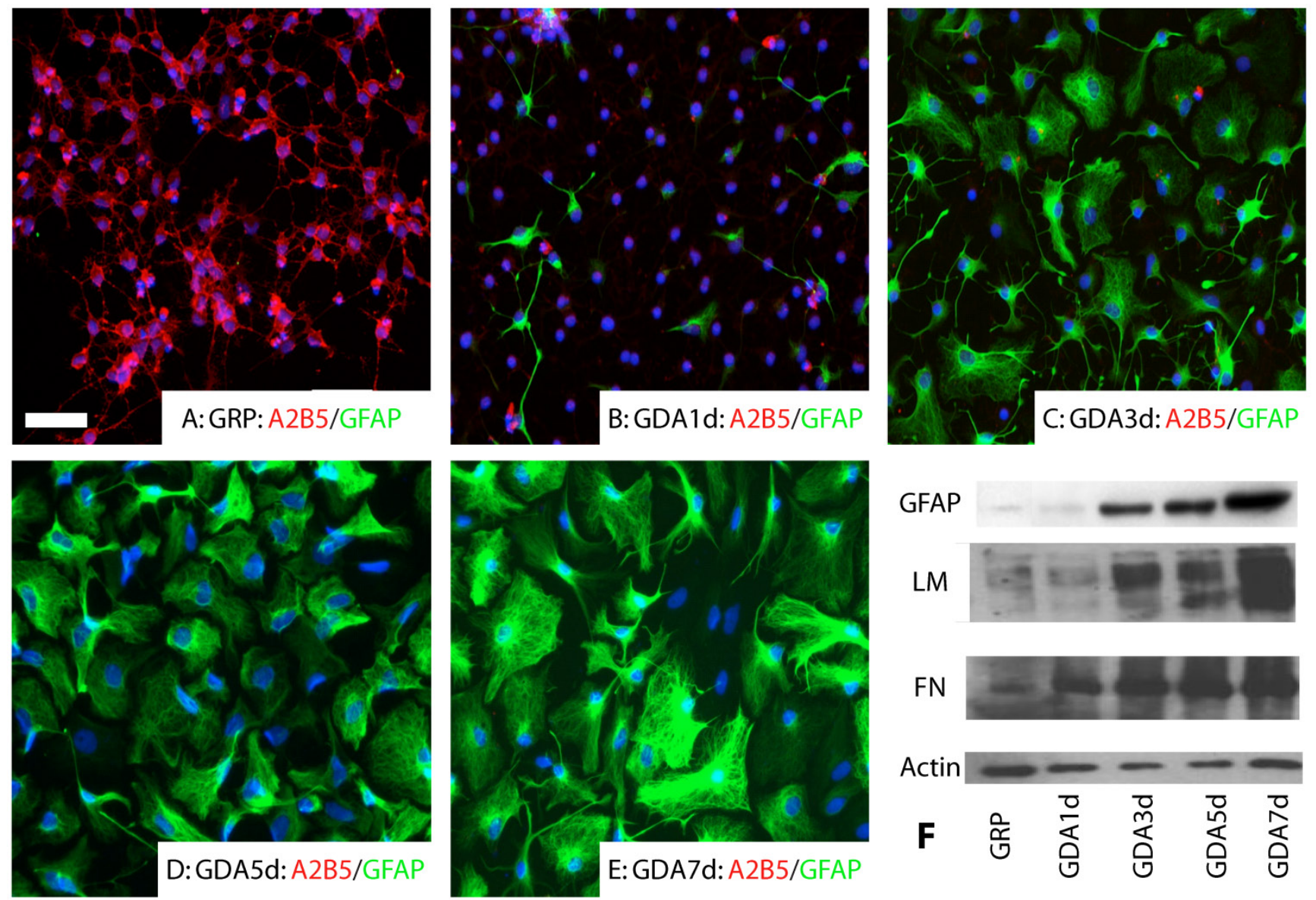

Figure I. Astrocyte differentiation of GRPs in vitro. (A) All undifferentiated GRPs expressed A2B5 but not GFAP. (B) After differentiated I day in the presence of BMP4, the expression of A2B5 was dramatically decreased and some cells started to express GFAP. (C, D, E) After differentiated 3 days (C), 5 days (D) or 7 days (E) in the presence of BMP4, almost all cells differentiated into GFAP positive astrocytes. Expression of GFAP continued to increase along the longer differentiation time. (F) The Western blot results confirmed that expression of GFAP, laminin (LM) and fibronectin (FN) was all increased with increasing differentiated time in the culture. Scale bar for A-E, $20 \mu \mathrm{m}$. 

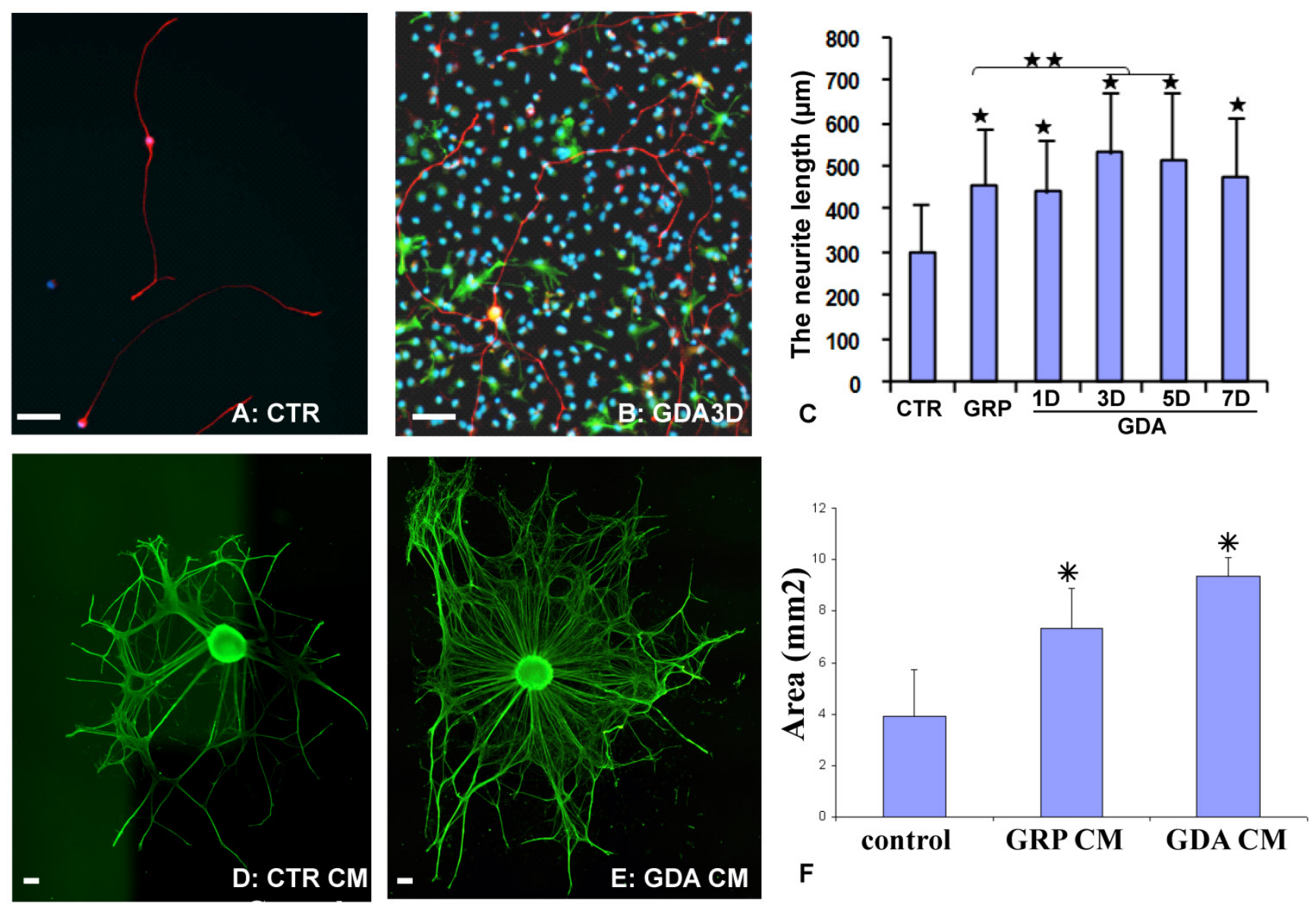

Figure 2. GDAs promoted axonal growth in vitro. (A) After been cultured for 18 hours in the control culture condition, most dorsal root ganglion neurons (DRGNs) were bipolar or unipolar without secondary branch from the primary processes. (B) After culture with GDAs for 18 hours, DRGNs were multipolar with more complex processes and secondary branches were often observed from the primary processes. (C) Quantitative analyses showed that the neurite length of DRGNs cultured in all cells was significantly longer than in control laminin $(p<0.05)$. The neurite length cultured in GDA3d or GDA5d was significantly longer than in GRPs, or GDAId ( $<<0.05$ ). (D) DRG explants cultured in control neurobasal medium had less neurite, viewed by NFM immunohistochemistry. (E) DRG explants cultured in GDA conditioned medium had much more neurite compared to the control. (F) Quantitative analyses showed that the neurite areas of DRG explants were significantly greater in GDA or GRP CMs than in control medium ( $P<0.05)$. Data in $C$ and $F$ represent the mean $\pm S D(n=4)$. Scale bars: $100 \mu \mathrm{m}$.

Figure 3. Survival of Grafted GDAs after SCl. Robust survival of grafted GDAs was observed in the injury epicenter. The grafted cells were mainly located in the spared white matter around the cavity with some within the cavity. The grafted GDAs were

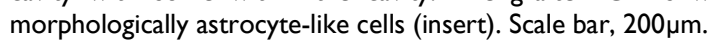

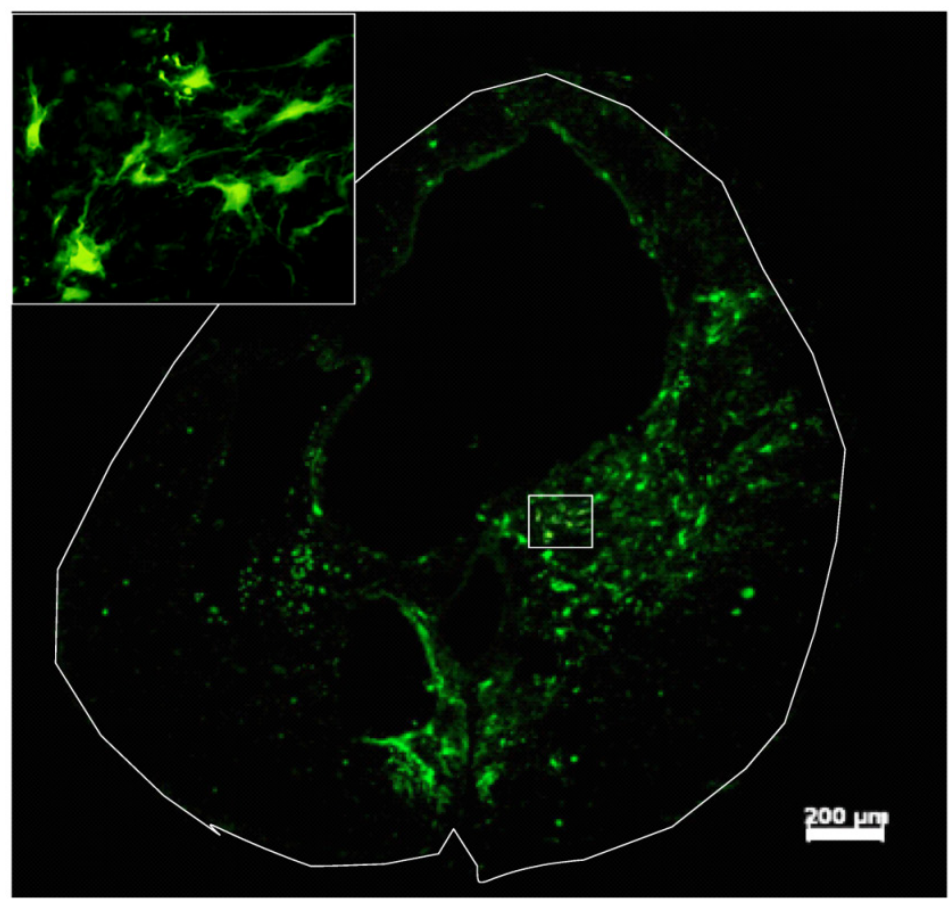



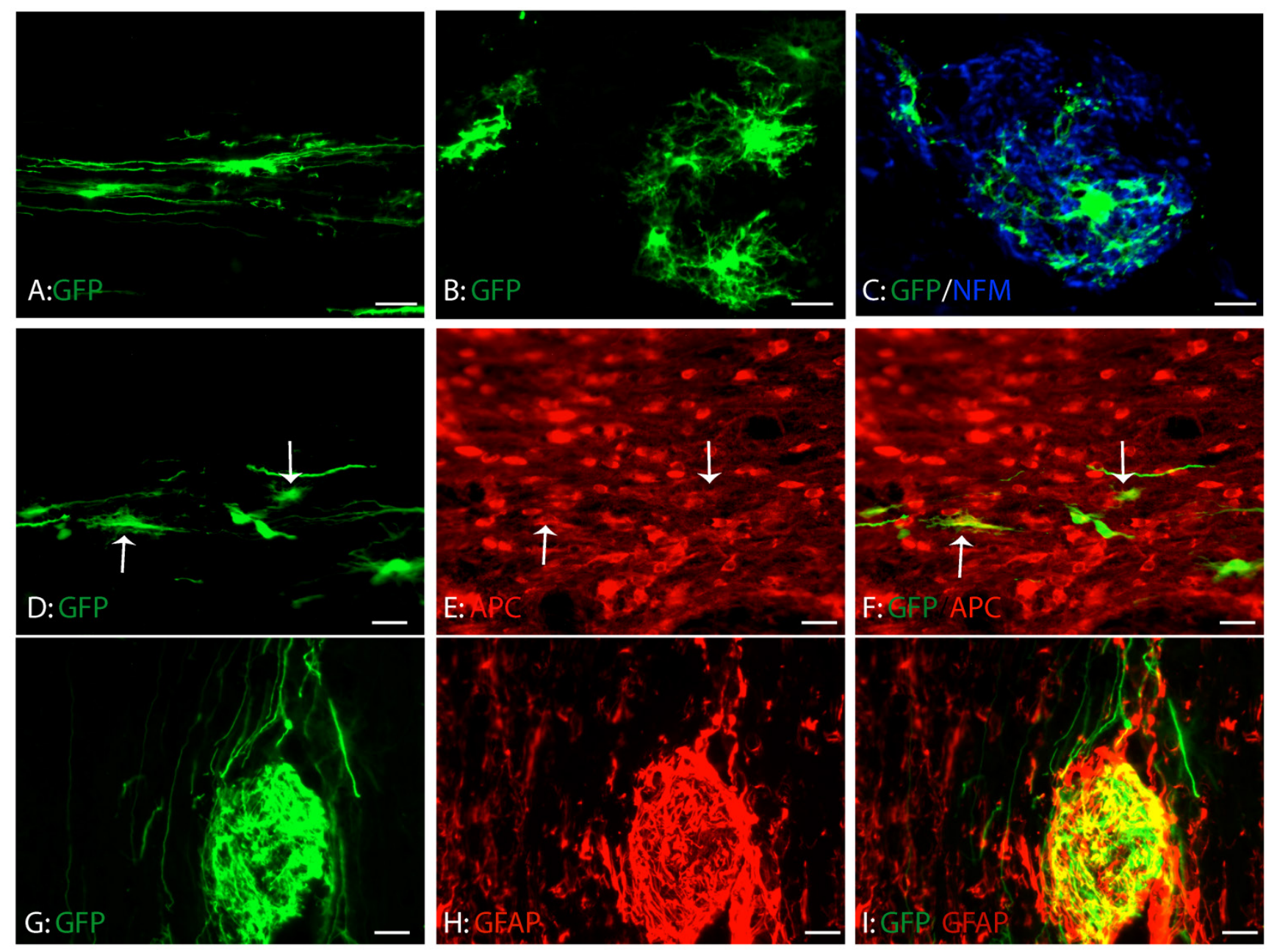

Figure 4. Differentiation of grafted GRPs after SCl. (A) Grafted GRPs survived and migrated in the white matter and sent multiple processes longitudinally. (B) The grafted GRPs differentiated into oligodendrocyte like cells with multiple processes. (C) The processes of oligodendrocyte like grafted GRPs formed close relationship with the host axons. (D-F) The grafted GRPs differentiated into oligodendrocytes expressing oligodendrocyte specific marker APC (arrows). (G-I) Many grafted GRPs also differentiated into GFAP positive astrocytes. Scare bars: $20 \mu \mathrm{m}$.

Almost all grafted GDAs were GFAP-positive astrocytes (Fig. 5A-C). No neuronal or oligodendrocyte differentiation was observed. These data showed that the grafted GDAs remained glial lineage. GDAs also mainly distributed and migrated in the white matter to form close relationship with the host axons (Fig. 5D-F). In the injured epicenter, the axons were observed to attach the grafted GDA bridges to across the injury epicenter (Fig. 5D-F).

D15A-GDA transplantation increased the spared white matter after SCI. Previous studies suggested that GRP or GDA transplantation could modify the injured environment to promote axonal regeneration [15]. To evaluate whether grafted GRPs or GDAs could modify gliosis after SCI, we double stained the injured spinal cord with GFAP and GFP. As shown in Fig. 5G-J, GFAP expression was significantly decreased in area receiving grafts of GRPs or GDAs. More importantly, CSPG expression was also significantly decreased in these areas with grafts of GDAs. The grafted GDAs were not expressed CSPG. In contrast, strong CSPG expression was observed in the host reactive astrocytes in the injured epicenter without GDA grafted (Fig. 5K-N). To test whether transplantation of GRPs or GDAs could promote axonal regeneration, we quantified the expression of GAP43 for the regenerating axons or expression of pan-axons for total axons in the injured epicenter. There was no significant difference in the expression of either GAP43, or pan-axons among all groups (data not shown). To further test whether transplantation of GRPs or GDAs could affect the spared white matter after SCI, we quantified the percentage of spared white matter to the total spinal cord at the injured epicenter as well as areas caudally or rostrally away from the epicenter. As shown in Fig 6 and 7, the percentage of spared white matter in epicenter, 0.6 or 1.2 $\mathrm{mm}$ caudally and rostrally was not significantly dif- 
ferent among control, GRP or GDA transplantation groups. However, the percentage of spared white matter in D15A-GDA group was significantly higher than other three groups in epicenter, $0.6 \mathrm{~mm}$ caudally and rostrally from the epicenter. These data showed that transplantation of D15A-expressing GDAs increased the spared white matter after SCI.
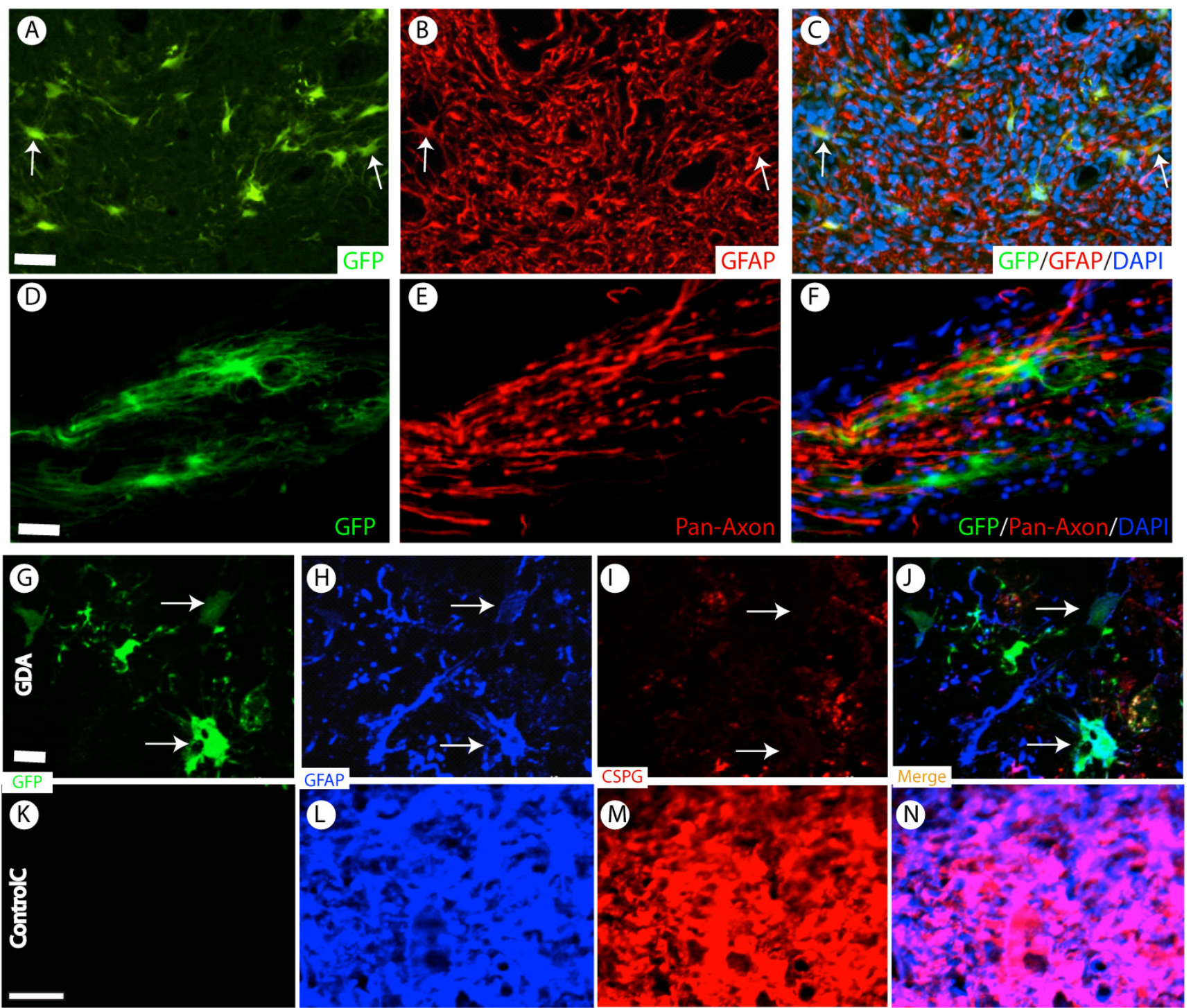

Figure 5. Survival and differentiation of grafted GDAs after SCI. (A-C) Almost all grafted GDAs (A, arrows) remained to express GFAP (B,C, arrows) at 2 months after transplantation. (D-F) The grafted GDAs sent multiple processes longitudinally to form cellular bridge for the host axons in the injured epi-center. (G-J) The grafted GDAs, which remained GFAP positive astrocytes ( $\mathrm{H}$, arrows), didn't express CSPG (I, arrows). The expression of CSPG (H) and GFAP (I) in the injured spinal cord was also dramatically decreased following transplantation of GDAs. (K-N) In contrast, in the injured epicenter without GDA graft, strong CSPG expression was observed in the host reactive astrocytes. Scale bars: $20 \mu \mathrm{m}$. 


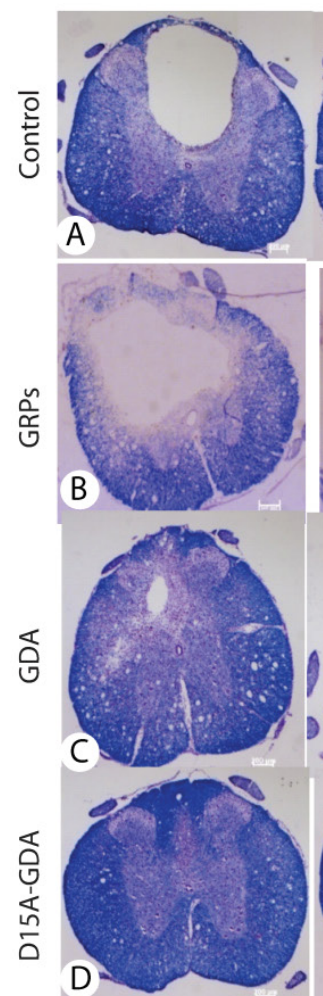

$\mathrm{C} 1.8 \mathrm{~mm}$
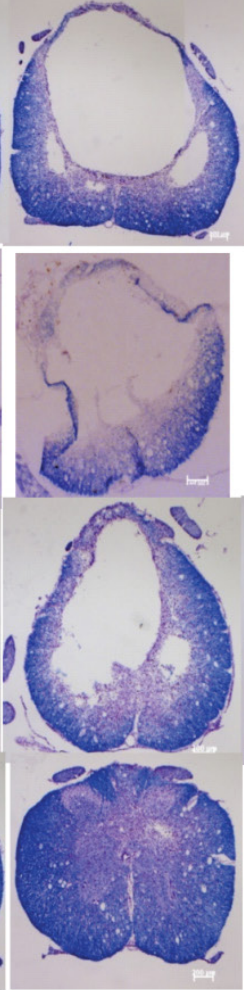

$\mathrm{C} 1.2 \mathrm{~mm}$
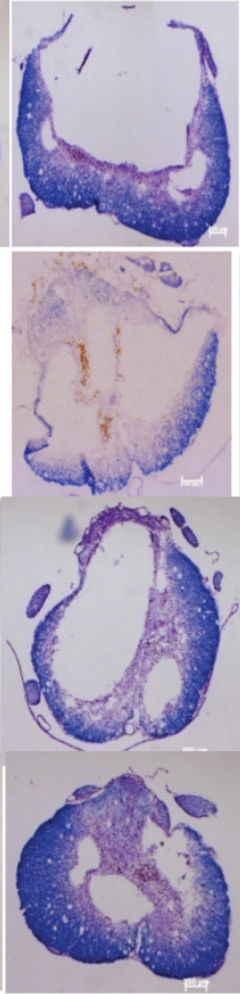

C0.6mm

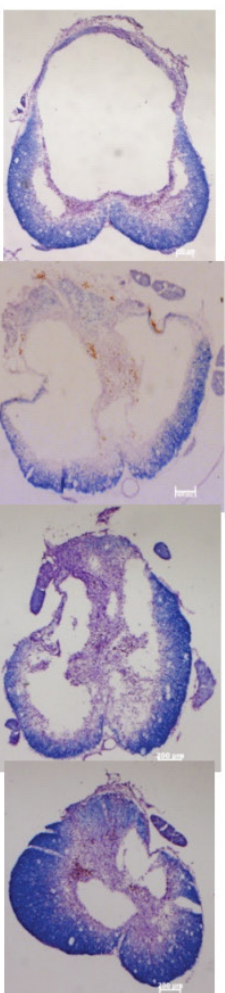

Epi-center
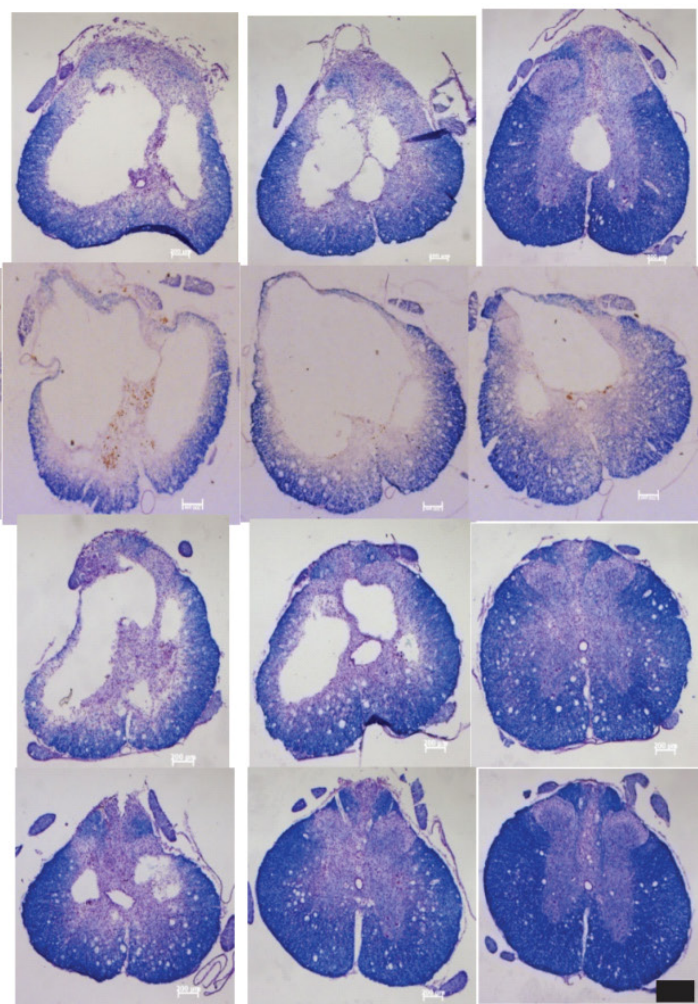

$\mathrm{R} 0.6 \mathrm{~mm}$

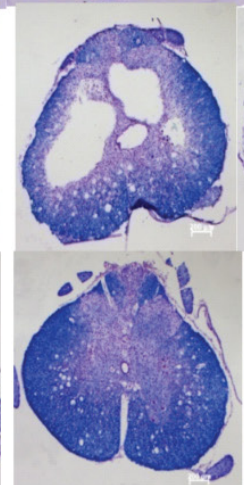

$\mathrm{R} 1.2 \mathrm{~mm}$

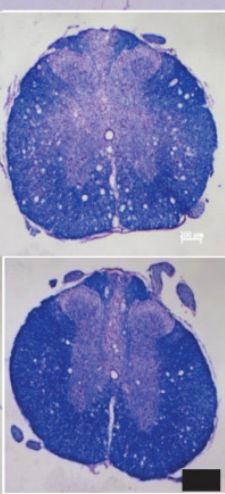

$\mathrm{R} 1.8 \mathrm{~mm}$

Figure 6. The injury cavity and spared white matter after $\mathrm{SCl}$ by the iron-eriochrom cyanine $\mathrm{R}(\mathrm{EC})$ staining. The injury size in control (A), GRP grafts (B), or GDA grafts (C) group was much bigger than in DI5A-GDA grafts group (D). The percentage of spared white, on the other hand, was significantly increased in DI5A-GDA group than in other three groups. Scale bars: $100 \mu \mathrm{m}$.

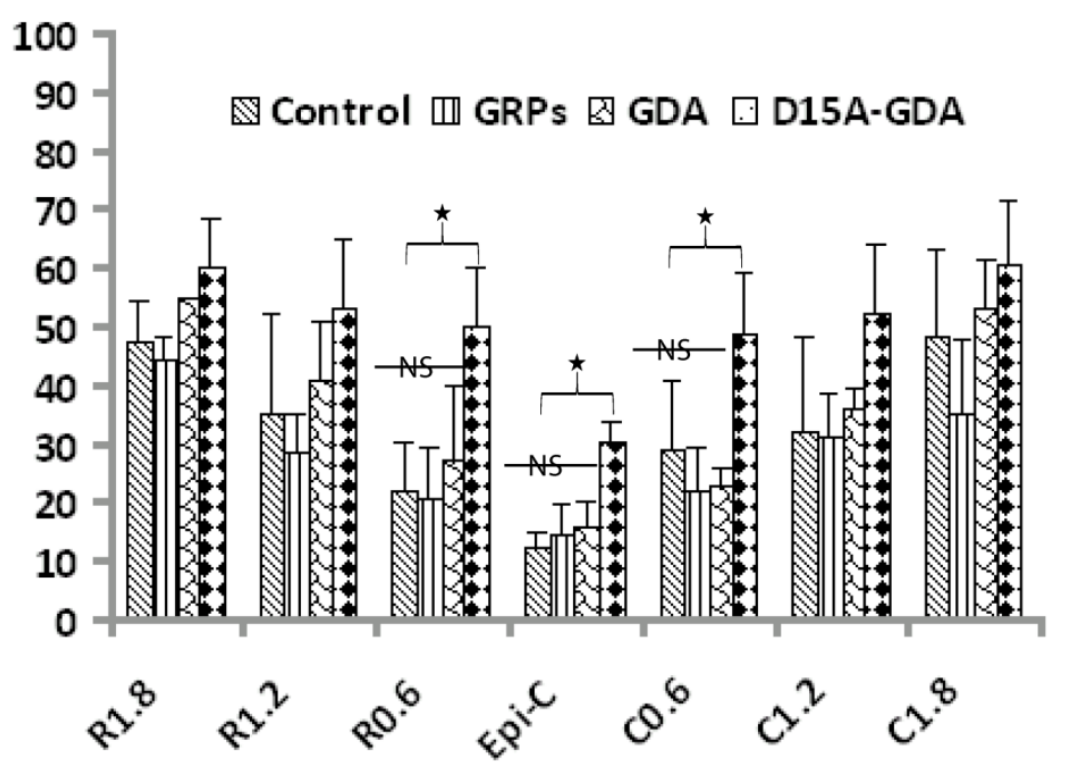

Figure 7. Increased spared white matter after SCl following DI5A-GDA transplantation. The percentages of spared white matter to the whole spinal cord were calculated and quantified using ES staining. The percentages of spared white matter in the injury epiecenter, $0.6 \mathrm{~mm}$ caudally or rostrally were significantly increased in DI5A-GDA grafted group compared to other three groups $(p<0.05)$. There was not significantly different among the control, GRP or GDA graft group. Data represent the mean \pm SD $(n=8)$. 
D15A-GDA transplantation increased the functional recovery after SCI. To test whether transplantation of GRPs or GDAs could promote functional recovery, we assessed BBB scores for the open-field locomotion and tradscan for foot-print analyses. The BBB scores were all significantly improved from the first weeks to 8 weeks after injury in all groups (Fig. $8 \mathrm{~A})$. This is consistent with previous studies in this model showing the spontaneous recovery of locomotion function after injury [37]. The BBB scores were not significantly different among all four groups at 1 week after injury before cell transplantation. After transplantation, the BBB scores in groups receiving grafts of GRPs or GDAs were also not significantly from the control groups at all tested time points from 1 to 8 weeks after transplantation. However, the BBB scores in D15A-GDAs graft group were significantly higher than other three groups from 1 week to 8 weeks after transplantation. The objective foot-print analyses also showed that the toe spreads in the injury
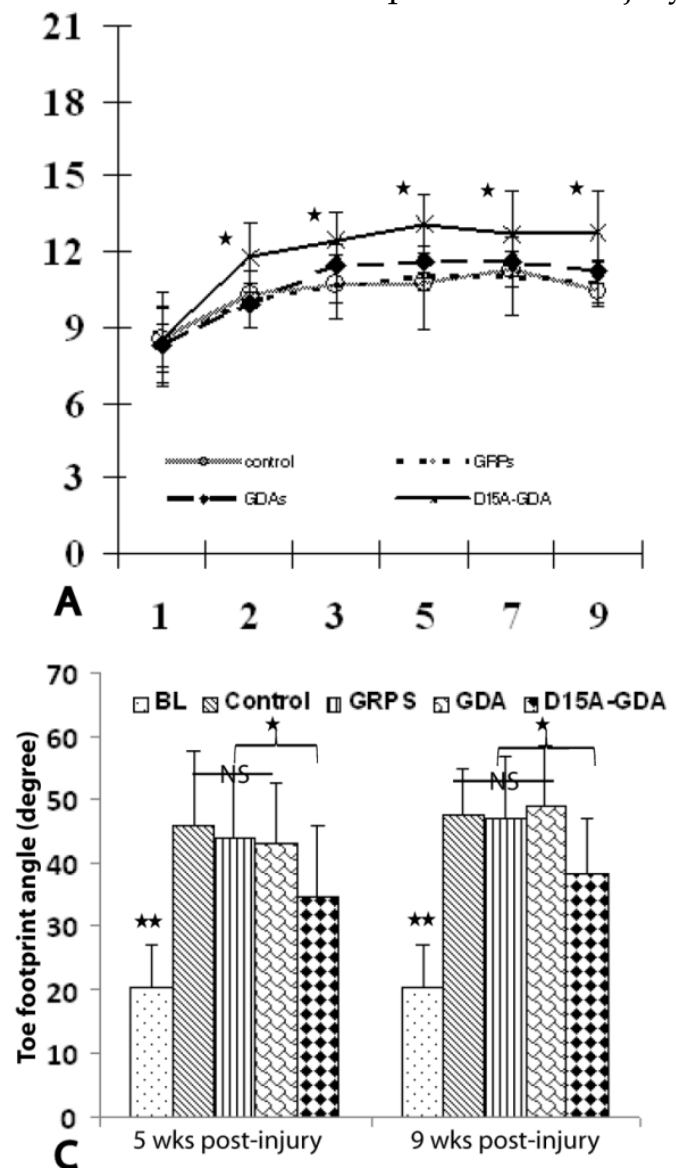

control, GRP or GDA graft group at 5 or 9 weeks after injury were significantly shorter compared to the baseline before injury (Fig. 8B). There was no significantly different among the control, GRP and GDA groups. However, the toe spreads in 15A-GDA graft group were significantly longer than the control, GRP or GDA group at both 5 and 9 weeks after injury. Similarly, the foot print angles were significantly increased in all injured groups compared to baseline before injury (Fig. 8C). There was no significant difference among the injury control, GRP and GDA groups at 5 and 9 weeks after injury. However, the foot print angles were significantly recovered in D15A-GDA group compared with the other groups at these time points (Fig. 8C). These results showed that transplantation of GRPs or GDAs alone failed to improve the locomotion function after SCI. But combination of GDA transplantation and D15A expression leaded to significantly functional recovery.
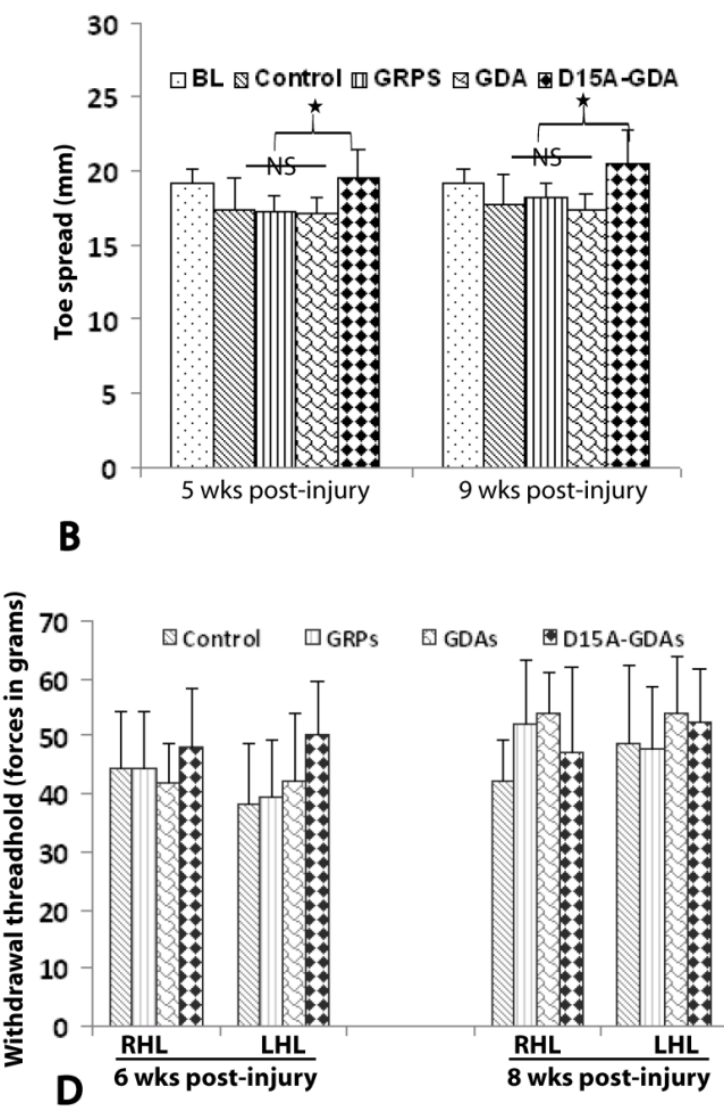

Figure 8. Locomotion recovery after DI5A-GDA transplantation. The locomotion functions were assessed by BBB locomotion score and treadscan footprint analyses. (A) BBB scores were not significantly different among all groups at I week post-injury (PI). From 2 week PI (I week after transplantation) to 9 weeks PI, BBB scores are significantly improved in DI5A-GDA group compared to control, GRP or GDA graft groups (P < 0.05 ). BBB scores were not significantly different among these three groups at all tested time points $(p>0.05)$. (B) Footprint analyses showed that toe spread measurements were significantly improved in DI5A-GDA graft group compared to control, GRP or GDA group at 5 or 9 weeks PI ( $<<0.05$ ). (C) Footprint analyses also showed that toe sprint angles were significantly larger in all grafted groups compared to baseline at 5 or 9 weeks $\mathrm{PI}$ ( $\mathrm{p}<0.05$ ). However, angles in DI5A-GDA group were significantly improved to become smaller compared to control, GRP, or GDA graft groups ( $P<0.05$ ). There was no significantly different among these three groups ( $p>0.05)$. (D) Von Frey hair monofilament test showed that the withdrawal forces were not significantly different among control, GRP, GDA or DI5A-GDA groups at both left and right limbs at 6 and 8 weeks PI $(p>0.05)$. Data in A-D represent the mean \pm SD ( $n=12$ ). 
Previous studies showed that transplantation of NSCs or GRPs could cause allodynia after SCI [16;41]. To determine if grafted GRPs or GDAs affected pain perception in transplanted animals, mechanical sensitivity (Von Frey test) was performed. Von Frey filament testing at both 6 and 8 weeks after injury (5 and 7 weeks after transplant) showed the paw withdrawal thresholds in GRP, GDA or D15A-GDA grafted animals were not significantly different from the injury control animals (Fig. 8D). These results suggested that transplantation of GRPs, GDAs or D15A-GDAs did not cause allodynia.

\section{Discussion}

In the present study, we have tested the therapeutic potential of GRPs and GDAs after contusion SCI. Robust survival and extensive migration of grafted GRPs were observed in the host injured spinal cord after transplantation. The grafted GRPs migrated and integrated in the host spinal cord. They differentiated into both astrocytes and oligodendrocytes. These results are consistent with previous in vitro [42] and in vivo [6;40] studies showing that GRPs have potential to differentiate into both astrocytes and OLs and could be one of the cell resources for astrocyte or oligodendrocyte replacement after SCI. Our results showed that GRPs or its conditioned media promoted neurite growth in vitro, suggesting that GRPs can promote axonal regeneration by providing substrates and/or secreting factors. This is consistent with previous study [43] showing GRPs enhance the axonal growth of co-cultured neurons even in the presence of CSPG. After transplantation into the injured spinal cord, GRPs also decreased the gliosis shown by the decrease of GFAP and CSPG expression in vivo. However, they did not promote significantly axonal regeneration and functional recovery. Our previous study [6] showed that transplantation of GRPs alone did not increase the spared white matter or functional recovery after contusion SCI. More recent studies also showed that transplantation of rat or human GRPs after contusion SCI improved the injured environment by reducing cyst and scar formation [44;45]. But neither rat nor human GRP grafts resulted in significant axonal regeneration and locomotion functional recovery. Similarly, transplantation of rat or human GRPs did not promote significant axonal regeneration and functional recovery after dorsolateral laceration of cervic spinal cord. These studies suggest that transplantation of GRPs alone may not be sufficient to promote significantly functional recovery after SCI although it shows some anatomical improvements. Combination of GRP transplantation with other treatments such as over-expression of multineuro- trophin D15A may be necessary for significantly functional recovery after SCI [6].

Although transplantation of undifferentiated GRPs fails to promote axonal regeneration and functional recovery after laceration SCI, priming GRPs into astrocytes before transplantation has proved effective to promote regeneration and functional recovery after dorsal hemisection SCI [15]. In this study, we extended to test the therapeutic potential of GDAs after contusion SCI. Our in vitro results showed that almost all GRPs differentiate into astrocytes after addition of BMP4 in the culture medium for 3 days. And the newly differentiated astrocytes from GRPs are more effective than undifferentiated GRPs to promote neurite growth of cultured DRGNs. Interestingly, the GRPs differentiated for 3 days are most effective and the ones differentiated for longer time such as 5 or 7 days are less effective. These results suggest that young GDAs could be more capable to promote axonal growth in vitro than the old ones. These results are consistent with previous studies using embryonic astrocytes. For example, embryonic astrocytes have long been thought of as an attractive cell type for repair of the adult CNS [46;47]. Astrocytes directly isolated from the embryonic CNS promote axonal regeneration after transplantation into adult $\mathrm{CNS}$ injuries [48;49]. But isolating embryonic astrocytes directly from the embryonic CNS is very challenging, owing to the relatively low abundance of these cells in vivo. The generation of postnatal astrocyte cultures is normally associated with prolonged growth in conditions in vitro that allow aging of these cells to a less supportive phenotype [50], which has also resulted in minimal axon growth after their transplantation to adult spinal cord injuries [51]. Our results suggest that young GDAs may mimic the astrocytes from developing CNS to support the axonal growth. Importantly, it is easy and fast to obtain a large, homogeneous population of embryonic-like supportive astrocytes from embryonic GRPs for transplantation [15;52].

We further tested the effects of GDAs on axonal regeneration and functional recovery after SCI. After transplantation, GDAs survived and integrated well into the injured host. They remained astrocyte lineage. In contrast to the host reactive astrocytes, the grafted GDAs did not express inhibitory CSPGs. Importantly, they formed close relationship with the host axons. In spite of its anatomical benefits, however, transplantation of GDAs alone did not lead to significant axonal regeneration shown by GAP 43 staining or locomotion functional recovery by BBB scores. These results are unexpected since our in vitro data show that GDAs or its conditioned medium can promote axonal or neurite growth in vitro. And pre- 
vious studies also showed that transplantation of GDAs into a hemisection SCI promoted robust axonal regeneration from rubrospinal axons and ascending sensory axons into and out of the lesion, resulting in functional recovery $[15 ; 53]$. However, there are a few differences between these studies and the present study. For example, they transplanted the GDAs immediately after injury. We grafted GDAs at 7 days after SCI. The injury models are also different between these studies. They used dorsal hemisection $\mathrm{SCI}$ and we used the contusion injury. Consistent with our present study, a recent study also showed that transplantation of human GDAs after contusion also resulted in some anatomical benefits but not functional recovery [44], suggesting that the injury model may play important roles in the in vivo functions of grafted GDAs. The microenvironment in the different injury models may be very different. Similarly, the microenvironments in different time points after injury are also very different. These different injury environments could induce the grafted GDAs to behave differently. Previous studies show that astroccytes derived from the same GRPs induced by BMPs or CNTF function very different after transplantation into the hemisection SCI [16;53]. The astrocytes derived from GRPs by BMPs show significant histology and locomotion functional benefits, but not allodynia. In contrast, astrocytes derived from GRPs by CNTF cause allodynia but not any histology or locomotion functional recoveries. These studies suggest that different astrocytes could function very differently after transplantation into the injured spinal cord. Future studies are needed to further determine whether GDAs become different types of astrocyte after transplanted into different injuries and how GDAs function differently in different injury environment.

Since transplantation of GDAs alone is not sufficient to allow significant funtional recovery after contusion SCI, we further tested whether combination of GDA transplantation and increasing expression of multi-neurotrophin, D15A, would lead to greater recovery. Our results showed that transplantation of D15A-expressing GDAs significantly increased the spared tissue and importantly, promoted the locomotion functional recovery. Our previous study showed that transplantation of D15A-expressing GRPs also promoted functional recovery [6]. There are a few differences between the present and our previous D15A-GRPs studies. GRPs differentiate into oligodendrocytes after transplantation in the contused spinal cord and expression of D15A doubles its oligodendrocyte differentiation. In contrast, grafted GDAs remain the astrocyte lineage without differentiation into oligodendrocytes. Secondly, D15A-GDA transplantation significantly increases the spared tissue after contusion SCI. In contrast, D15A-GRP transplantation fails to do so. Thirdly, the locomotion functional recovery in D15A-GDA transplantation is much earlier: one week after D15A-GDA transplantation compared to five weeks after D15A-GRP transplantation. Thus, functional recovery after D15A-GRPs transplantation is at least partially due to its increasing remyelination. However, the functional recovery after D15A-GDA transplantation may come from the mechanisms other than remyelination since D15A-GDAs lost it potential to differentiate into oligodendrocytes. Although the underneath mechanisms for functional recovery after D15A-GDA transplantation need to be further studied, our results, such as significant increase in spared tissue and early functional recovery, suggest that neuroprotection could be one of the important mechanisms. Consistent with our results, previous studies demonstrated that delivery of neurotrophin NT3 or BDNF with or without grafted cells was one of effective approaches to promote neuroprotection after SCI [54;55]. In addition, enhancing axonal sprouting and regeneration [12;22;28] or endogenous remyelination [18] by D15A-expressing GDAs may also contribute to the functional recovery, especially in the chronic SCI. However, it is difficult to study its effects on axonal regeneration and sprouting in the incomplete contusion injury since the spared and regenerated axons are not easy to distinguish in this injury model. It is worthy to note that our previous study [6] show delivery of multineurotrophin D15A alone does not lead to significant locomotion functional recovery after contusion SCI. The functional recovery after transplantation of D15A-expressing GDAs is likely the synergistic benefits from both GDAs and D15A expression.

Multiple different types of cells have been tested in combination with neurotrophin after SCI [56]. For example, Schwann cell transplantation combining with neurotrophin delivery promotes axonal regeneration and Schwann cell remyelination after SCI [57;58]. Neurotrophin-expressing bone mesenchymal stem cells (BMSC) show the benefits to bridge the injury and promote axonal sprouting and regeneration after transplantation [30]. Similarly, olfactory ensheathing cells (OECs) or fibroblasts also show behavioral or histological benefits after combination with neurotrophins following SCI [59]. Compared to these cells, GRPs and GDAs are origin cells of central nervous system and integrate into the glial injured environment much better after transplantation. GDAs provide additional benefits to decrease the glial scare in addition to provide a cellular bridge or scaffold for axonal regeneration $[15 ; 53]$. GDAs also create a better 
glial microenvironment to support the survival of host neurons [60;61] or the co-grafted neuron precursor cells [62]. Schwann cells, BMSC and even OECs have the benefit for autologous transplantation. This is important for clinical translation since autologous transplantation will obviate the need for immunosuppression and avoid the ethical issues using abortion fetus tissue or human ESCs. Nonetheless, the newly developed induced pluripotent stem cells technology $[63 ; 64]$ offers the potential for autologous GRPs and GDAs for treatment of SCI and other neurological diseases.

Concern about neuropathic pain has been raised in previous studies using neural stem cell transplantation after contusion [41] or GRP graft following dorsal hemisection SCI [16]. To address this important concern, we also test whether pain is increased after transplantation of GRPs or GDAs following contusion SCI. Our results showed that GRP or GDA grafts did not increase pain even though astrocyte differentiation from transplanted GRPs and GDA is present at and around the injury site. This result is contrast to previous studies [16;53], which showed that transplantation of GRPs or GDA differentiated by CNTF but not GDA differentiated by BMP increased allodynia after hemisection SCI. As discussed previously, there are a few differences among the present study and their studies, the different injured models (contusion versus hemisection) and different transplantation time after injury (1w after SCI versus immediate after hemisection). These differences especially the different injured SCI models could be one of the reasons for the contrast allodynia results between the present study and the previous studies [16]. In fact, previous studies showed that transplantation of human GRPs [44] or rat GRPs [45] after contusion SCI did not cause allodynia. These results are consistent with our present study, suggesting that not all astrocytes derived from transplanted neural stem cells or glial precursor cells will cause allodynia or increase neuropathic pain. The differentiation of grafted GRPs may be different in the contusion or hemisection SCI. Different subtypes of astroyctes could cause opposite effects on pain after transplantation into the injured spinal cord [16].

In summary, we showed that grafted GRPs or GDAs survived and integrated into the injured spinal cord. They improved the anatomical outcomes by decreasing gliosis, but did not promote functional recovery. However, combination of transplantation of GDAs and delivery of multi-neurotrophin D15A significantly increased the spared white matter and promoted locomotion functional recovery. These results suggest that combination of GDA transplanta- tion with neurotrophin expression may be an effective therapeutic approach for SCI.

\section{Acknowledgements}

This research was supported NS061975 (QLC) and The TIRR Foundation through Mission Connect (QLC).

\section{Competing Interests}

The authors have declared that no competing interest exists.

\section{References}

1. Karimi-Abdolrezaee S, Eftekharpour E, Wang J, Morshead CM, Fehlings MG. Delayed transplantation of adult neural precursor cells promotes remyelination and functional neurological recovery after spinal cord injury. J Neurosci 2006; 26(13):3377-3389.

2. Cummings BJ, Uchida N, Tamaki SJ, Salazar DL, Hooshmand M, Summers $\mathrm{R}$ et al. Human neural stem cells differentiate and promote locomotor recovery in spinal cord-injured mice. Proc Natl Acad Sci U S A 2005; 102(39):14069-14074.

3. McDonald JW, Liu XZ, Qu Y, Liu S, Mickey SK, Turetsky D et al. Transplanted embryonic stem cells survive, differentiate and promote recovery in injured rat spinal cord. Nat Med 1999; 5(12):1410-1412.

4. Keirstead HS, Nistor G, Bernal G, Totoiu M, Cloutier F, Sharp K et al. Human embryonic stem cell-derived oligodendrocyte progenitor cell transplants remyelinate and restore locomotion after spinal cord injury. J Neurosci 2005; 25(19):4694-4705.

5. Sharp J, Frame J, Siegenthaler M, Nistor G, Keirstead HS. Human embryonic stem cell-derived oligodendrocyte progenitor cell transplants improve recovery after cervical spinal cord injury. Stem Cells 2010; 28(1):152-163

6. Cao Q, Xu XM, DeVries WH, Enzmann GU, Ping P, Tsoulfas P et al. Functional recovery in traumatic spinal cord injury after transplantation of multineurotrophin-expressing glial-restricted precursor cells. J Neurosci 2005; 25(30):6947-6957.

7. Cao Q, He Q, Wang Y, Cheng X, Howard RM, Zhang $Y$ et al. Transplantation of ciliary neurotrophic factor-expressing adult oligodendrocyte precursor cells promotes remyelination and functional recovery after spinal cord injury. J Neurosci 2010; 30(8):2989-3001.

8. Bambakidis NC, Miller $\mathrm{RH}$. Transplantation of oligodendrocyte precursors and sonic hedgehog results in improved function and white matter sparing in the spinal cords of adult rats after contusion. Spine J 2004; 4(1):16-26.

9. Cao QL, Howard RM, Dennison JB, Whittemore SR. Differentiation of engrafted neuronal-restricted precursor cells is inhibited in the traumatically injured spinal cord. Exp Neurol 2002; 177(2):349-359.

10. Han SS, Kang DY, Mujtaba T, Rao MS, Fischer I. Grafted lineage-restricted precursors differentiate exclusively into neurons in the adult spinal cord. Exp Neurol 2002; 177(2):360-375.

11. Bonner JF, Blesch A, Neuhuber B, Fischer I. Promoting directional axon growth from neural progenitors grafted into the injured spinal cord. J Neurosci Res 2010; 88(6):1182-1192.

12. Lu P, Jones LL, Snyder EY, Tuszynski MH. Neural stem cells constitutively secrete neurotrophic factors and promote extensive host axonal growth after spinal cord injury. Exp Neurol 2003; 181(2):115-129.

13. Zhang YW, Denham J, Thies RS. Oligodendrocyte progenitor cells derived from human embryonic stem cells express neurotrophic factors. Stem Cells Dev 2006; 15(6):943-952.

14. Rossi SL, Nistor G, Wyatt T, Yin HZ, Poole AJ, Weiss JH et al. Histological and functional benefit following transplantation of motor neuron progenitors to the injured rat spinal cord. PLoS One 2010; 5(7):e11852.

15. Davies JE, Huang C, Proschel C, Noble M, Mayer-Proschel M, Davies SJ. Astrocytes derived from glial-restricted precursors promote spinal cord repair. J Biol 2006; 5(3):7.

16. Davies JE, Proschel C, Zhang N, Noble M, Mayer-Proschel M, Davies SJ. Transplanted astrocytes derived from BMP- or CNTF-treated glial-restricted precursors have opposite effects on recovery and allodynia after spinal cord injury. J Biol 2008; 7(7):24. 
17. Hattiangady B, Shuai B, Cai J, Coksaygan T, Rao MS, Shetty AK. Increased dentate neurogenesis after grafting of glial restricted progenitors or neural stem cells in the aging hippocampus. Stem Cells 2007; 25(8):2104-2117.

18. McTigue DM, Horner PJ, Stokes BT, Gage FH. Neurotrophin-3 and brain-derived neurotrophic factor induce oligodendrocyte proliferation and myelination of regenerating axons in the contused adult rat spinal cord. J Neurosci 1998; 18(14):5354-5365.

19. Nakahara Y, Gage FH, Tuszynski MH. Grafts of fibroblasts genetically modified to secrete NGF, BDNF, NT-3, or basic FGF elicit differential responses in the adult spinal cord. Cell Transplant 1996; 5(2):191-204.

20. Ye JH, Houle JD. Treatment of the chronically injured spinal cord with neurotrophic factors can promote axonal regeneration from supraspinal neurons. Exp Neurol 1997; 143(1):70-81.

21. Onifer SM, Magnuson DSK, Shield CB, Whittemore SR. Grafting into the injured spinal cord to restore locomotion: two decades of promise yet to be fulfilled. NeuroScience News 2000; 3(6):50-58.

22. Grill R, Murai K, Blesch A, Gage FH, Tuszynski MH. Cellular delivery of neurotrophin-3 promotes corticospinal axonal growth and partial functional recovery after spinal cord injury. J Neurosci 1997; 17(14):5560-5572.

23. Blits B, Dijkhuizen PA, Boer GJ, Verhaagen J. Intercostal nerve implants transduced with an adenoviral vector encoding neurotrophin-3 promote regrowth of injured rat corticospinal tract fibers and improve hindlimb function. Exp Neurol 2000; 164(1):25-37.

24. Houweling DA, Lankhorst AJ, Gispen WH, Bar PR, Joosten EA. Collagen containing neurotrophin-3 (NT-3) attracts regrowing injured corticospinal axons in the adult rat spinal cord and promotes partial functional recovery. Exp Neurol 1998; 153(1):49-59.

25. Lu P, Yang H, Jones LL, Filbin MT, Tuszynski MH. Combinatorial therapy with neurotrophins and cAMP promotes axonal regeneration beyond sites of spinal cord injury. J Neurosci 2004; 24(28):6402-6409.

26. Bradbury EJ, Khemani S, Von R, King, Priestley JV, McMahon SB. NT-3 promotes growth of lesioned adult rat sensory axons ascending in the dorsal columns of the spinal cord. Eur J Neurosci 1999; 11(11):3873-3883.

27. Oudega M, Hagg T. Neurotrophins promote regeneration of sensory axons in the adult rat spinal cord. Brain Res 1999; 818(2):431-438.

28. Jin Y, Fischer I, Tessler A, Houle JD. Transplants of fibroblasts genetically modified to express BDNF promote axonal regeneration from supraspinal neurons following chronic spinal cord injury. Exp Neurol 2002; 177(1):265-275.

29. Jin Y, Tessler A, Fischer I, Houle JD. Fibroblasts genetically modified to produce BDNF support regrowth of chronically injured serotonergic axons. Neurorehabil Neural Repair 2000; 14(4):311-317.

30. Lu P, Jones LL, Tuszynski MH. BDNF-expressing marrow stromal cells support extensive axonal growth at sites of spinal cord injury. Exp Neurol 2005; 191(2):344-360.

31. Urfer R, Tsoulfas P, Soppet D, Escandon E, Parada LF, Presta LG. The binding epitopes of neurotrophin-3 to its receptors trkC and gp75 and the design of a multifunctional human neurotrophin. EMBO J 1994; 13(24):5896-5909.

32. Mayer-Proschel M, Kalyani AJ, Mujtaba T, Rao MS. Isolation of lineage-restricted neuronal precursors from multipotent neuroepithelial stem cells. Neuron 1997; 19(4):773-785.

33. Mujtaba T, Piper DR, Kalyani A, Groves AK, Lucero MT, Rao MS. Lineage-restricted neural precursors can be isolated from both the mouse neural tube and cultured ES cells. Dev Biol 1999; 214(1):113-127.

34. Kinsella TM, Nolan GP. Episomal vectors rapidly and stably produce high-titer recombinant retrovirus. Hum Gene Ther 1996; 7(12):1405-1413.

35. Cao Q, Zhang YP, Iannotti C, DeVries WH, Xu XM, Shields CB et al. Functional and electrophysiological changes after graded traumatic spinal cord injury in adult rat. Exp Neurol 2005; 191 Suppl 1:S3-S16.

36. Cao QL, Zhang YP, Howard RM, Walters WM, Tsoulfas P, Whittemore SR. Pluripotent stem cells engrafted into the normal or lesioned adult rat spinal cord are restricted to a glial lineage. Exp Neurol 2001; 167(1):48-58.

37. Zhang YP, Burke DA, Shields LB, Chekmenev SY, Dincman T, Zhang Y et al. Spinal cord contusion based on precise vertebral stabilization and tissue displacement measured by combined assessment to discriminate small functional differences. J Neurotrauma 2008; 25(10):1227-1240.

38. Basso DM, Beattie MS, Bresnahan JC. A sensitive and reliable locomotor rating scale for open field testing in rats. J Neurotrauma 1995; 12(1):1-21.

39. Oorschot DE. Are you using neuronal densities, synaptic densities or neurochemical densities as your definitive data? There is a better way to go. Prog Neurobiol 1994; 44(3):233-247.

40. Han SS, Liu Y, Tyler-Polsz C, Rao MS, Fischer I. Transplantation of glial-restricted precursor cells into the adult spinal cord: survival, glial-specific differentiation, and preferential migration in white matter. Glia 2004; 45(1):1-16.

41. Hofstetter CP, Holmstrom NA, Lilja JA, Schweinhardt P, Hao J, Spenger $\mathrm{C}$ et al. Allodynia limits the usefulness of intraspinal neural stem cell grafts; directed differentiation improves outcome. Nat Neurosci 2005; 8(3):346-353.

42. Mujtaba T, Mayer-Proschel M, Rao MS. A common neural progenitor for the CNS and PNS. Dev Biol 1998; 200(1):1-15.

43. Ketschek AR, Haas C, Gallo G, Fischer I. The roles of neuronal and glial precursors in overcoming chondroitin sulfate proteoglycan inhibition. Exp Neurol 2012; 235(2):627-637.

44. Jin Y, Neuhuber B, Singh A, Bouyer J, Lepore A, Bonner J et al. Transplantation of human glial restricted progenitors and derived astrocytes into a contusion model of spinal cord injury. J Neurotrauma 2011; 28(4):579-594.

45. Nout YS, Culp E, Schmidt MH, Tovar CA, Proschel C, Mayer-Proschel M et al. Glial restricted precursor cell transplant with cyclic adenosine monophosphate improved some autonomic functions but resulted in a reduced graft size after spinal cord contusion injury in rats. Exp Neurol 2011; 227(1):159-171.

46. Silver J. Transplantation strategies using embryonic astroglial cells to promote CNS axon regeneration in neonatal and adult mammals. Clin Res 1988; 36(3):196-199.

47. Smith GM, Miller RH, Silver J. Astrocyte transplantation induces callosal regeneration in postnatal acallosal mice. Ann N Y Acad Sci 1987; 495:185-206.

48. Kliot M, Smith GM, Siegal JD, Silver J. Astrocyte-polymer implants promote regeneration of dorsal root fibers into the adult mammalian spinal cord. Exp Neurol 1990; 109(1):57-69.

49. Wunderlich G, Stichel CC, Schroeder WO, Muller HW. Transplants of immature astrocytes promote axonal regeneration in the adult rat brain. Glia 1994; 10(1):49-58.

50. Smith GM, Rutishauser U, Silver J, Miller RH. Maturation of astrocytes in vitro alters the extent and molecular basis of neurite outgrowth. Dev Biol 1990; 138(2):377-390.

51. Wang JJ, Chuah MI, Yew DT, Leung PC, Tsang DS. Effects of astrocyte implantation into the hemisected adult rat spinal cord. Neuroscience 1995; 65(4):973-981.

52. Rao MS, Noble M, Mayer-Proschel M. A tripotential glial precursor cell is present in the developing spinal cord. Proc Natl Acad Sci U S A 1998; 95(7):3996-4001.

53. Davies SJ, Shih CH, Noble M, Mayer-Proschel M, Davies JE, Proschel C. Transplantation of specific human astrocytes promotes functional recovery after spinal cord injury. PLoS One 2011; 6(3):e17328.

54. Liu Y, Himes BT, Murray M, Tessler A, Fischer I. Grafts of BDNF-producing fibroblasts rescue axotomized rubrospinal neurons and prevent their atrophy. Exp Neurol 2002; 178(2):150-164.

55. Kwon BK, Liu J, Lam C, Plunet W, Oschipok LW, Hauswirth W et al. Brain-derived neurotrophic factor gene transfer with adeno-associated viral and lentiviral vectors prevents rubrospinal neuronal atrophy and stimulates regeneration-associated gene expression after acute cervical spinal cord injury. Spine (Phila Pa 1976 ) 2007; 32(11):1164-1173.

56. Tetzlaff W, Okon EB, Karimi-Abdolrezaee S, Hill CE, Sparling JS, Plemel JR et al. A systematic review of cellular transplantation therapies for spinal cord injury. J Neurotrauma 2011; 28(8):1611-1682.

57. Xu XM, Guenard V, Kleitman N, Aebischer P, Bunge MB. A combination of BDNF and NT-3 promotes supraspinal axonal regeneration into Schwann cell grafts in adult rat thoracic spinal cord. Exp Neurol 1995; 134(2):261-272.

58. Deng LX, Hu J, Liu N, Wang X, Smith GM, Wen X et al. GDNF modifies reactive astrogliosis allowing robust axonal regeneration through Schwann cell-seeded guidance channels after spinal cord injury. Exp Neurol 2011; 229(2):238-250.

59. Ma YH, Zhang Y, Cao L, Su JC, Wang ZW, Xu AB et al. Effect of neurotrophin-3 genetically modified olfactory ensheathing cells transplantation on spinal cord injury. Cell Transplant 2010; 19(2):167-177.

60. Lepore AC, Rauck B, Dejea C, Pardo AC, Rao MS, Rothstein JD et al. Focal transplantation-based astrocyte replacement is neuroprotective in a model of motor neuron disease. Nat Neurosci 2008; 11(11):1294-1301.

61. Lepore AC, O'Donnell J, Kim AS, Williams T, Tuteja A, Rao MS et al. Human glial-restricted progenitor transplantation into cervical spinal cord of the SOD1 mouse model of ALS. PLoS One 2011; 6(10):e25968.

62. Bonner JF, Connors TM, Silverman WF, Kowalski DP, Lemay MA, Fischer I. Grafted neural progenitors integrate and restore synaptic connectivity across the injured spinal cord. J Neurosci 2011; 31(12):4675-4686. 
63. Takahashi K, Tanabe K, Ohnuki M, Narita M, Ichisaka T, Tomoda K et al. Induction of pluripotent stem cells from adult human fibroblasts by defined factors. Cell 2007; 131(5):861-872.

64. Yu J, Vodyanik MA, Smuga-Otto K, Antosiewicz-Bourget J, Frane JL, Tian $S$ et al. Induced pluripotent stem cell lines derived from human somatic cells. Science 2007; 318(5858):1917-1920. 